\title{
Jefferson
}

Thomas Jefferson University

$\overline{\text { HOME OF SIDNEY KIMMEL MEDICAL COLLEGE }}$

JHN Journal

Volume 14

Issue 1 Summer 2019

Article 5

Summer 2019

\section{Spinal Cord Stimulation in the 21st Century - Reviewing Innovation in Neuromodulation}

\author{
Victor Sabourin, MD \\ Thomas Jefferson University \\ Justin Turpin, BA \\ Thomas Jefferson University \\ Jeffery Head, BA \\ Thomas Jefferson University \\ Chengyuan Wu, MD \\ Thomas Jefferson University Hospital \\ Ashwini Sharan, MD \\ Thomas Jefferson University \\ Follow this and additional works at: https://jdc.jefferson.edu/jhnj

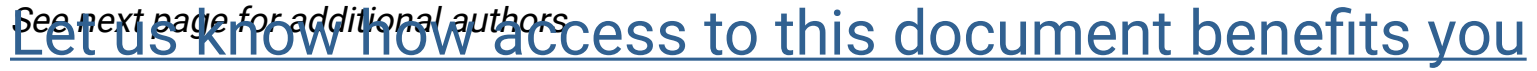

\section{Recommended Citation}

Sabourin, MD, Victor; Turpin, BA, Justin; Head, BA, Jeffery; Wu, MD, Chengyuan; Sharan, MD, Ashwini; and Hoelscher, MD, Christian (2019) "Spinal Cord Stimulation in the 21st Century - Reviewing Innovation in Neuromodulation," JHN Journal: Vol. 14 : Iss. 1 , Article 5.

DOI: https://doi.org/10.29046/JHNJ.014.1.005

Available at: https://jdc.jefferson.edu/jhnj/vol14/iss1/5

This Article is brought to you for free and open access by the Jefferson Digital Commons. The Jefferson Digital Commons is a service of Thomas Jefferson University's Center for Teaching and Learning (CTL). The Commons is a showcase for Jefferson books and journals, peer-reviewed scholarly publications, unique historical collections from the University archives, and teaching tools. The Jefferson Digital Commons allows researchers and interested readers anywhere in the world to learn about and keep up to date with Jefferson scholarship. This article has been accepted for inclusion in JHN Journal by an authorized administrator of the Jefferson Digital Commons. For more information, please contact: JeffersonDigitalCommons@jefferson.edu. 


\section{Spinal Cord Stimulation in the 21st Century - Reviewing Innovation in Neuromodulation}

\section{Authors}

Victor Sabourin, MD; Justin Turpin, BA; Jeffery Head, BA; Chengyuan Wu, MD; Ashwini Sharan, MD; and Christian Hoelscher, MD 


\section{Spinal Cord Stimulation in the 21st Century-Reviewing Innovation in Neuromodulation}

\author{
Victor Sabourin, $\mathrm{MD}^{1}$; Justin Turpin, $\mathrm{BA}^{2}$; Jeffrey Head, $\mathrm{BA}^{2}$; Chengyuan $\mathrm{Wu}, \mathrm{MD}^{1}$; \\ Ashwini Sharan, $M D^{1}$; Christian Hoelscher, $M D^{1}$ \\ ${ }^{1}$ Department of Neurological Surgery, Thomas Jefferson University, Philadelphia, PA, 19107 \\ 2 Sidney Kimmel Medical College, Thomas Jefferson University, Philadelphia, PA, 19107
}

\section{INTRODUCTION}

Low back pain (LBP) is a pervasive problem impacting health systems across the world. In the United States, chronic LBP impacts up to $40 \%$ of Americans and results in excessive financial strain on the healthcare budget, estimated at up to $\$ 100$ billion annually. ${ }^{1}$ Furthermore, treatment results are often disappointing, with the traditional pathway of conservative measures, narcotic pain medication, and surgical decompression and/or fusion leading to both patient and provider frustration, complications, and diminished patient productivity and quality of life. This has naturally led to questions from policymakers regarding the utility of healthcare dollars spent on back pain. In this milieu, a variety of neuromodulation techniques have found a niche in the management of this patient population, with indications commonly quoted including failed back surgery syndrome (FBSS), chronic neuropathic pain, and complex regional pain syndrome (CRPS), among others. ${ }^{1,2}$ From its inception on the basis of Melzak and Wall's gate theory ${ }^{3}$, to its first human trial in the $1960 \mathrm{~s},{ }^{4}$ and to the modern era, spinal cord stimulation has undergone a series of innovations that have expanded indications and improved patient outcomes. The goal of this study is to summarize the most important clinical trials involving both traditional SCS and newer stimulation paradigms to provide an overview of the current state of affairs of this rapidly-growing field.

\section{METHODS}

We performed a PubMed literature search utilizing the following terms: SCS, spinal cord stimulation, neuromodulation, high frequency stimulation, paresthesia free, HF10, failed back surgery syndrome, and chronic pain. Only English language articles were reviewed. All prospective, randomized controlled trials pertaining to the use of neuromodulation in the treatment of chronic back and limb pain were included. The data extraction was performed by three reviewers $(\mathrm{JT}, \mathrm{JH}, \mathrm{CH})$, and reviewed by the senior author (CW). The selected studies were analyzed, and relevant results were summarized as follows.

\section{RESULTS}

\section{Traditional, Low Frequency, Tonic SCS}

Several landmark trials paved the way for the widespread use of spinal cord stimulation in the treatment of chronic back and limb pain. These early studies utilized low frequency stimulation generally in the $40-100 \mathrm{~Hz}$ range delivered in a tonic manner, producing paresthesias that overlap the areas where the patient experiences pain. North et al5 randomized 51 FBSS patients with chronic lower limb pain with or without back pain to initial treatment with a low frequency stimulator or re-operation. The primary endpoint was "success", defined as $>50 \%$ reported pain relief and patient satisfaction with treatment at 2-years post-operatively or at last follow-up. Secondary end points included treatment crossover, success at last follow-up, and improvement in medication use, daily activities, and neurologic status. At mean three-year follow-up, "success" was achieved in a significantly higher proportion of patients randomized to SCS (47\%) compared to those randomized to reoperation. ${ }^{5,6}$ These findings remained statistically significant even after worst-case analysis which assumed patients unavailable for long-term follow-up in the SCS group were all treatment failures. Further, a significantly higher proportion of patients in the reoperation arm crossed over to SCS $(54 \%)$ compared to only $21 \%$ of SCS patients who elected to undergo reoperation. While patient reported functional capacity didn't reach a significant difference, SCS patients did require significantly fewer opiate equivalents for pain control. Kumar et al ${ }^{7,8}$ followed this up with the PROCESS study which randomized 100 FBSS patients with limb>back pain to SCS with medical management $(n=52)$ or medical management alone $(n=48)$. The primary end point of the study was defined as $>50 \%$ relief of leg pain, with secondary end points including quality of life, functional capacity as measured by the Oswestry Disability Index (ODI), patient satisfaction, and changes in pain medication usage. At 6-month followup, the primary endpoint was achieved in $48 \%$ of SCS patients compared to $9 \%$ in the medical management alone group. The SCS group also reported significantly greater quality of life, improvement in ODI, treatment satisfaction, and reduced back pain compared to medical management alone. SCS patients were also more likely to reduce drug intake based on morphine equivalents as well as decrease use of nondrug therapies. Similar improvements were maintained at 12 and 24 month follow-up analyses. ${ }^{7.8}$

\section{Paresthesia-Free SCS}

As clinical experience with traditional, paresthesia-based systems grew, interest began to develop in creating new stimulation protocols that would generate pain relief without the need for paresthesia overlap as a significant number of patients found these sensations to be uncomfortable, particularly when there 
is significant postural variation which can make certain daily activities, such as driving, difficult or painful. Furthermore, traditional spinal cord stimulation, while relatively successful at treating appendicular neuropathic symptoms, struggled with relief of more nociceptive axial pain where adequate paresthesia overlap is difficult to achieve. Given how common low back pain is in the general population, a more efficient means for targeting this symptom complex was needed. Buyten et $\mathrm{al}^{9}$ prospectively enrolled 82 patients with back pain with or without associated leg pain in a trial of high frequency, low amplitude, paresthesia free stimulation using the Nevro device. The outcomes of interest were VAS scores, ODI, sleep disturbances per night, and patient satisfaction. The trial to conversion rate was $88 \%$. VAS back and leg scores improved at 6 months, ${ }^{8.4}$ to 2.7 for back and 5.4 to 1.4 for leg pain. ODI improved by 17 points and patients reported 2.4 fewer episodes of sleep disturbance per night. Similar results were obtained with HF10 therapy in another prospective observational study in patients with a primary complaint low back pain, with back pain and leg pain reduced by $61 \%$ and $58 \%$, respectively, based on $\mathrm{VAS}^{10}$. While promising, these and other observational studies did not provide a control group to compare against. To that end, De Andres et $\mathrm{al}^{11}$ compared high frequency $(10 \mathrm{kHz})$ stimulation to conventional stimulation, randomizing 55 patients with FBSS with neuropathic back or leg pain. The results suggested no significant difference at one year, with both stimulation profiles showing significant benefit compared to baseline values. ${ }^{11}$ On the other hand, North et al, ${ }^{12}$ in their pilot study randomizing patients with FBSS who had a previously implanted, paresthesia-based system with inadequate pain relief, noted that pain relief via NRS and disability via ODI were significantly improved with $1 \mathrm{kHz}$ stimulation. ${ }^{12}$ Perruchoud and colleagues ${ }^{13}$ similarly randomized patients with chronic neuropathic back and leg pain, previously stable on a conventional SCS system, to periods of sham and $5 \mathrm{kHz}$, subthreshold tonic stimulation. The periods of sham and $5 \mathrm{kHz}$ stimulation were separated by a "washout" period during which the patients reverted to their traditional, paresthesia-based pattern. The primary outcome was the Patient's Global Impression of Change (PGIC), with secondary outcomes including VAS and the Euroqol questionnaire EQ-5D. At the end of the study, the authors noted no difference between sham and high-frequency stimulation, with what appeared to be a "period effect" in that patients tended to respond more favorably to sham or $5 \mathrm{kHz}$ stimulation based purely on which was initiated first and responded less favorably to whichever pattern was tested second. However, the comparison to baseline values is confusing and again the use of previously stabilized tSCS patients is a variable that must be considered. The most robust data in this field came from Kapural and colleagues who explored the ability of low amplitude, high frequency spinal cord stimulation at $10 \mathrm{kHz}$ to provide durable relief of both axial and appendicular pain in the SENZA trial. ${ }^{14,15}$ In this prospective, multicenter, randomized trial, the investigators randomized 198 patients with medically refractory back and leg pain to either high frequency stimulation (HF10) or traditional low frequency, paresthesia-dependent treatment (tSCS). The primary endpoint was $>50 \%$ reduction on the visual analog scale (VAS), with secondary outcomes including opioid use, functional disability as measured by ODI, and percentage change from baseline back and leg symptoms. A total of 171 patients had positive trials and were ultimately implanted (HF10, $n=90$; tSCS, $\mathrm{n}=81$ ). At the initial 3-month evaluation, $84.5 \%$ of HF10 patients achieved the primary endpoint for back pain, compared to only $43.8 \%$ of tSCS patients. Similarly, for leg pain HF10 success on the primary endpoint was $83.1 \%$ compared to $55.5 \%$ for tSCS. These values remained similar at 12-month follow-up evaluation, with remission rates (VAS <2.5) for back and leg pain approaching $67 \%$ for HF10, compared to $35-40 \%$ for tSCS. At 24-month evaluation, the difference in primary endpoint success was still maintained in favor of HF10 for both back and leg pain $(76.5 \%$ and $72.9 \%$ respectively for HF10, compared to $50 \%$ for tSCS). Secondary outcome analysis also favored HF10, with greater overall percentage reduction of back and leg pain compared to baseline, higher likelihood of achieving minimal disability on ODI, and higher patient reported treatment satisfaction. Additionally, 11.3\% of patients in the tSCS group reported uncomfortable paresthesias, with no patients in the HF10 group reporting any paresthesia-related issues. ${ }^{14,15}$

Despite accumulating clinical evidence regarding the efficacy of high frequency SCS, several questions remained. One such issue is the impact of varying frequencies and other stimulation parameters on treatment effect. One study ${ }^{16}$ prospectively randomized a cohort of 24 patients into a blinded crossover study, with each patient experiencing 3 weeks at a time of sham stimulation, and stimulation at $1200 \mathrm{~Hz}$, $3030 \mathrm{~Hz}$, and $5882 \mathrm{~Hz}$. The devices were programmed such that amplitude was maintained slightly below threshold level, unique to each patient and frequency. The primary outcome was the reduction of VAS back pain scores. Baseline VAS was reported at 7.75, with improvement to $4.83,4.51,4.57$, and 3.22 for the trial groups (sham, $1200 \mathrm{~Hz}, 3030 \mathrm{~Hz}$, and $5882 \mathrm{~Hz}$, respectively). All comparisons to baseline were significant, but within the treatment groups only $5882 \mathrm{~Hz}$ had a significantly greater impact on outcome. The authors argued that while designed as a study on frequency, the impact of pulse width on allowable amplitude without generating paresthesia yielded higher charger-per-second dosing with higher frequency stimulation, which may have played a role in the results. The results also questioned to what degree pain relief afforded by spinal cord stimulation is a result of placebo, as at the end of the study $12.5 \%$ of patients preferred the sham stimulation protocol. A similar study also explored the impact of varying degrees of high-frequency stimulation on treatment effect, noting the unclear mechanism of HF10 and the unclear impact of frequency on clinical outcome. In this study, Thomson et $\mathrm{al}^{17}$ randomized 21 patients with chronic back>leg pain who had passed a trial of $10 \mathrm{kHz}$ stimulation and were implanted with permanent devices. Each patient experienced four weeks of stimulation, in random order, at $10 \mathrm{kHz}$, as well as 1-, 4-, and $7 \mathrm{kHz}$. At each frequency, pulse width and amplitude were adjusted to optimize therapy. The impact on the 
primary outcome, NRS for back, leg, and overall pain, was similar between groups with all frequency groups showing about $50 \%$ reduction in each category. There were no between-group differences. There was significantly less charge delivered in the $1 \mathrm{kHz}$ stimulation than in the other three groups. Interestingly, the calculated charge delivered per second showed a non-linear relationship with frequency, suggesting that frequency modification in isolation may not deliver appropriate symptom relief, highlighting the importance of the interplay between frequency, pulse width, and amplitude.

As interest in paresthesia-free stimulation grows, other investigators are exploring novel stimulation protocols. Burst stimulation, a technique based on short intervals of high-frequency, low amplitude stimulation followed by periods of inactivity, is one such protocol thought to work in at least two mechanisms: 1) more closely mimic neuronal firing in the central nervous system with impacts on higher-order thalamo-cingulate pathways, and 2) provide inhibition of $A \delta$ and $c$ fibers via subthreshold antidromic $A \beta$ activation with resultant activation of inhibitory interneurons. De Ridder et al18 performed an early trial with this technology, randomizing 15 patients undergoing a trial of spinal cord stimulation to 7 days each of burst, tonic, and sham stimulation. Primary endpoints included VAS for back, limb, and general pain, with secondary endpoints including the pain vigilance and awareness scale (PVAQ), and worst/best pain levels during a given trial week. They noted that burst stimulation did not induce more paresthesias than sham stimulation. The primary outcome measure showed significant improvements in back, limb, and general pain comparing burst stimulation to placebo. Because burst stimulation produced no noticeable paresthesias, this marked the first time in a randomized trial that spinal cord stimulation could be proven better than control/sham treatment, an important landmark in SCS research. Not surprisingly, tSCS showed significant improvement in limb and general pain, but not back pain, compared to placebo. Comparing burst to tSCS, the mean change in back pain favored burst ( -3.8 on VAS compared to -2.2), but this did not reach significance. Limb pain between the two protocols was the same (both -3.9 on VAS). General pain was significantly reduced with burst therapy (-4.5 VAS) compared to tSCS (-2.5). Regarding the PVAQ, tonic and placebo stimulation showed no impact on attention to pain or attention to changes in pain, whereas burst stimulation significantly improved these parameters, suggesting an impact on affective and attentional components of pain. Schu et al ${ }^{19}$ compared high frequency stimulation to a burst protocol, randomizing 20 patients with FBSS and a previously implanted, burst-capable system to separate, one week periods of placebo stimulation, 500 $\mathrm{Hz}$ tonic stimulation, and burst stimulation (5 pulses at $500 \mathrm{~Hz}$, delivered 40 times per second), with the primary outcome of interest the impact on the numerical rating scale (NRS) for pain intensity. Baseline values were obtained prior to entering the protocol, with the device programmed for standard, paresthesia-based stimulation. While burst stimulation was the only pattern to significantly reduce the NRS score, the magnitude of the treatment effect was modest (5.6 at baseline, 4.7 for burst stimulation), and disability as measured by ODI showed only a nonsignificant decrease. However, it is worth reiterating that the "control" values in this study were based on patients stabilized on a paresthesia-based system, and thus treatment effects can be expected to be blunted, and the fact that at the end of the trial $80 \%$ of the patients preferred the burst protocol is also significant. More recently, Deer et $\mathrm{a}^{20}$ published results of the SUNBURST trial, which randomized patients with refractory back and leg pain to tSCS or burst stimulation. The trial was conducted in two phases. First patients were randomized to a 12 -week period of a given stimulation treatment, and then switched to the other stimulation method for the next 12 weeks. Thereafter, the patients were allowed to choose their preferred stimulation method and were assessed every 6 months for two years. The primary endpoint was change in VAS. A total of 100 patients were randomized, with 45 entering tSCS first followed by burst, and 55 vice versa. At 12- and 24-week analyses, both non-inferiority and superiority of burst stimulation as compared to tSCS were established via the primary endpoint of VAS reduction. Secondary end point analyses revealed that $61.6 \%$ of patients were paresthesia-free with burst compared to only $2.7 \%$ of tSCS patients. While $78.1 \%$ of patients were satisfied overall with both stimulation methods, $70.8 \%$ reported preferring burst stimulation with lack of paresthesia being the most common reason cited. This preference was maintained at oneyear follow-up with $68.2 \%$ of patients continuing to prefer burst therapy. Much like high frequency stimulation, as the clinical efficacy became clear, more questions emerged regarding mechanisms and the impact of various stimulation parameters. One study ${ }^{21}$ randomized 15 patients previously implanted with a burst-capable device in the setting of FBSS to one of two stimulation patterns: 1) 5 pulses delivered at $500 \mathrm{~Hz}$ with a $1000 \mu \mathrm{sec}$ pulse width, 40 times per second or 2) 5 pulses delivered at $1000 \mathrm{~Hz}, 1000$ Hsec pulse width, 40 times per second. The amplitude, and thus the total electrical dose delivered remained the same. Clinical outcomes were not significantly different.

\section{DISCUSSION}

Spinal cord stimulation was first put into clinical practice in the 1960s for an attempt at palliation in a patient with terminal cancer pain. ${ }^{4}$ The momentum behind its initial development was the ubiquitous Gate Control Theory put forth by Melzack and Wall. ${ }^{3}$ While generally accepted that this theory is overly simplistic regarding the mechanism of action in the various spinal cord stimulation techniques, it did inspire generations of physicians and scientists to develop new means of tackling medically refractory chronic pain syndromes. ${ }^{22}$ Traditional, lowfrequency, suprathreshold tonic spinal cord stimulation has been postulated to work via several mechanisms. Most commonly cited includes selective activation of large, myelinated $A \beta$ fibers with subsequent inhibition of smaller, pain-mediating $A \delta$ and $c$ fibers via inhibitory interneurons. Other postulated contributors include dorsal horn wide dynamic range neurons, thought to develop a hypersensitivity in neuropathic injury states with resultant increased basal glutamate release and subsequent glutamate:GABA imbalance. Supraspinal mechanisms are also thought to be at play although the exact brainstem-spinal 
circuitry activated by spinal cord stimulation remains largely theoretical. ${ }^{22}$ Regardless of the inner workings of the therapy, spinal cord stimulation has proven to be an important development in the management of an otherwise frustrating patient population. Medical management in chronic pain conditions often fails and is associated with high rates of narcotic medication use with their associated complications. Futile treatment regimens, although inexpensive in isolation, become expensive when applied indefinitely, and costly revision surgeries without clearly rectifiable structural or compressive pathology are not only low yield, but very expensive and potentially dangerous. Spinal cord stimulation has provided an opportunity to attain symptom relief, limit disability, and improve patient productivity. And although the upfront investment is large, there is an increasing amount of evidence suggesting that the long-term cost-effectiveness profile is positive and that spinal cord stimulation should be considered earlier in the broader treatment paradigm for chronic pain. ${ }^{1,2}$ With all of this said, spinal cord stimulation is certainly no panacea. One of the most important limiting factors is the requirement for a high-degree of overlap between induced paresthesia and the patient's baseline pain. This becomes a problem for patients that can't tolerate even mild paresthesia. Furthermore, positional differences in the intensity of elicited paresthesia have been reported by some patients, and occasionally even limit participation in important day-to-day activities such as driving. The requirement for paresthesia overlap also makes the treatment of axial symptoms particularly challenging, as effective paresthesia coverage in this region remains elusive even with today's technology. Given that chronic back pain is one of the most common problems in modern medical systems, achieving efficient low back coverage is a priority for next generation models.

With these challenges in mind, innovation in spinal cord stimulation has moved towards paresthesia-free systems. Burst patterns were developed as a means of subthreshold stimulation that would more closely resemble central nervous system neuronal firing. Furthermore, simultaneous SCS/EEG studies have suggested that burst stimulation impacts cortical medial pain pathways, with De Ridder et al ${ }^{18}$ showing an effect on connectivity involving dorsolateral prefrontal, dorsal anterior cingulate, and parahippocampal regions. In other words, burst stimulation may impact affective and attentional components to pain in addition to pain transmission. High-frequency, low-amplitude stimulation patterns have been developed ranging up to $10,000 \mathrm{~Hz}$. Again, the exact mechanism of action of these systems remains elusive, but several hypotheses have been put forth, including production of a local sodium channel blockade with inhibition of depolarization within the dorsal columns, desynchronization of neuronal messaging from the periphery, and inhibition of wide dynamic range neuronal sensitization, among others. ${ }^{22}$ What is clearer is that the clinical benefit has been demonstrated in several randomized controlled trials, most notably the SENZA tria ${ }^{14,15}$ where both axial and limb pain response rates approached $70 \%$ up to two years after implantation. From a surgical perspective, the purely anatomic placement of HF10 leads creates additional benefit in terms of ease of placement for the surgeon, without the need for uncomfortable wakeup testing for the patient and likely shorter overall anesthesia time. However, such systems are not without their limits, as well. There is a certain subset of patients that prefer paresthesias, ostensibly as a reassuring cue that there is in fact a therapy being provided. Furthermore, very high frequency systems such as HF10 deliver much more charge per second, which can lead to short internal pulse generator (IPG) lifetime, or in the case of rechargeable IPGs, significant charging burden for the patient. Additionally, the exact impact of frequency, amplitude, and pulse width, and thus total charge delivery, are unclear and have not been systematically accounted for in the available literature. It is highly likely that simple frequency modulation is not by itself responsible for the clinical benefit of this technique, and a clearer understanding of how these parameters come together for therapeutic effect will be critical if we are to maximize the potential benefit.

\section{CONCLUSION}

Chronic back pain remains a highly prevalent clinical problem in modern society. This patient population has historically been very challenging to treat. Spinal cord stimulation has helped to bridge the treatment gap in these patients, and while progress so far has been encouraging, there remains much research to be done to fully understand the mechanisms and potential therapeutic reach of this modality.

\section{REFERENCES}

1. Hoelscher C, Riley J, Wu C, Sharan A. CostEffectiveness Data Regarding Spinal Cord Stimulation for Low Back Pain. Spine. 2017 Jul 15;42 Suppl 14:S72-S79

2. Sharan A, Riley J, Hoelscher C. An Overview of Chronic Spinal Pain: Revisiting Diagnostic Categories and Exploring an Evolving Role for Neurostimulation. Spine. 2017 Jul 15;42 Suppl $14: S 35-40$

3. Melzack R, Wall P. Pain Mechanisms: A New Theory. Science 1965;150:971-9

4. Shealy C, Mortimer J, Reswick J. Electrical Inhibition of Pain By Stimulation of the Dorsal Columns: Prelminary Clinical Report. Anesth Analg. 1967;46:489-91.

5. North R, Kidd D, Lee M, Piantodosi S. A prospective, randomized study of spinal cord stimulation versus reoperation for failed back surgery syndrome: initial results. Stereotactic and Functional Neurosurgery. 1994;62(14):267-272

6. North RB, Kidd DH, Farrokhi F, Piantadosi SA. Spinal cord stimulation versus repeated lumbosacral spine surgery for chronic pain: a randomized, controlled trial. Neurosurgery. 2005;56(1):98-106.

7. Kumar K, Taylor R, Jacques L, Eldabe S, Meglio M, Molet J, Thomson S, O'Callaghan J, Eisenberg E, Milbouw G, Buchser E, Fortini G, Richardson J, North R. Spinal cord stimulation versus conventional medical management for neuropathic pain: a multicentre randomised controlled trial in patients with failed back surgery syndrome. Pain. Nov 2007;132(12):179-188.

8. Kumar K, Taylor R, Jacques L, Eldabe S, Meglio M, Molet J, Thomson S, O'Callaghan J, Eisenberg E, Milbouw G, Buchser E, Fortini G, Richardson J, North R. The effects of spinal cord stimulation in neuropathic pain are sustained: a 24-month follow-up of the prospective randomized controlled multicenter trial of the effectiveness of spinal cord stimulation. Neurosurgery. Oct 2008;63(4):762-770 
9. Van Buyten J, Al-Kaisy A, Smet I, Palmisani S, Smith T. High-Frequency Spinal Cord Stimulation for the Treatment of Chronic Back Pain Patients: Results of a Prospective Multicenter European Clinical Study. Neuromodulation. 2013 Jan-Feb;16(1):59-5

10. Al-Kaisy A, Van Buyten J, Smet I, Palmisani S, Pang D, Smith T. Sustained Effectiveness of $10 \mathrm{kHz}$ High-Frequency Spinal Cord Stimulation for Patients with Chronic, Low Back Pain: 24-Month Results of a Prospective Multicenter Study. Pain Med. 2014 Mar;15(3):347-54

11. De Andres J, Monsalve-Doiz V, Fabregat-Cid G, Vallanueva-Perez V, Harutyunyan A, Asensio-Samper J, Sanchis-Lopez N. Prospective, Randomized Blind Effect-onOutcome Study of Conventional vs HighFrequency Spinal Cord Stimulation in Patients with Pain and Disability Due to Failed Back Surgery Syndrome. Pain Med. 2017 Dec 1;18(12):2401-2421

12. North J, Hong K, Cho P. Clinical Outcomes of $1 \mathrm{kHz}$ Subperception Spinal Cord Stimulation in Implanted Patients With Failed Paresthesia-Based Stimulation: Results of a Prospective Randomized Controlled Trial. Neuromodulation. 2016 Oct;19(7):731-737.

13. Perruchoud C, Eldabe S, Batterham A, Madzinga G, Brookes M, Durrer A, Rosato M, Boet N, West S, Bovy M, Rutschmann B, Gulve A, Garner F, Buchser E. Analgesic Efficacy of High-Frequency Spinal Cord Stimulation: A Randomized Double-Blind PlaceboControlled Study. Neuromodulation. 2013 Jul-Aug;16(4):363-9.
14. Kapural L, Yu C, Doust M, Gliner B, Vallejo R, Sitzman B, Amirdelfan K, Morgan D, Brown L, Yearwood T, Bundschu R, Burton A, Yang $\mathrm{T}$, Benyamin R, Burgher A. Novel $10-\mathrm{kHz}$ High-Frequency Therapy (HF10 Therapy) Is Superior to Traditional Low-Frequency Spinal Cord Stimulation for the Treatment of Chronic Back and Leg Pain: The SENZA-RCT Randomized Controlled Trial. Anesthesiology 2015 Oct:123(4):851-60.

15. Kapural L, Yu C, Doust M, Gliner B, Vallejo R, Sitzman B, Amirdelfan K, Morgan D, Yearwood $\mathrm{T}$, Bundschu R, Yang T, Benyamin R, Burgher A. Comparison of 10-kHz High-Frequency and Traditional Low-Frequency Spinal Cord Stimulation for the Treatment of Chronic Back and Leg Pain: 24-Month Results From a Multicenter, Randomized, Controlled Pivotal Trial. Neurosurgery. 2016 Nov;79(5)j:667-677.

16. Al-Kaisy A, Palmisani S, Pang D, Sanderson K, Wesley S, Tan Y, McCammon S, Trescott A. Prospective, Randomized, Sham-Control, Double Blind, Crossover Trial of Subthreshold Spinal Cord Stimulation at Various Kilohertz Frequencies in Subjects Suffering From Failed Back Surgery Syndrome (SCS Frequency Study). Neuromodulation. 2018 Jul;21(5):457-465.

17. Thomson S, Tavakkolizadeh M, Love-Jones S, Patel N, Gu J, Bains A, Doan Q, Moffitt M. Effects of Rate on Analgesia in Kilohertz Frequency Spinal Cord Stimulation: Results of the PROCO Randomized Controlled Trial. Neuromodulation. 2018 Jan;21(1):67-76.
18. De Ridder D, Plazier M, Kamerling N Menovsky T, Vanneste S. Burst spinal cord stimulation for limb and back pain. World Neurosurgery. Nov 2013;80(5):642-649 e641.

19. Schu S, Slotty PJ, Bara G, von Knop M, Edgar $D$, Vesper J. A prospective, randomised, double-blind, placebo-controlled study to examine the effectiveness of burst spinal cord stimulation patterns for the treatment of failed back surgery syndrome. Neuromodulation. Jul 2014;17(5):443-450.

20. Deer T, Slavin KV, Amirdelfan K, et al. Success Using Neuromodulation With BURST (SUNBURST) Study: Results From a Prospective, Randomized Controlled Trial Using a Novel Burst Waveform. Neuromodulation. Jan 2018;21(1):56-66.

21. Van Havenbergh T, Vancamp T, Van Looy P, Vanneste S, De Ridder D. Spinal Cord Stimulation for the Treatment of Chronic Back Pain Patients: $500-\mathrm{Hz}$ vs. $1000-\mathrm{Hz}$ Burst Stimulation. Neuromodulation. 2015 Jan ;18(1):9-12

22. Linderoth B, Foreman R. Conventional and Novel Spinal Stimulation Algorithms: Hypothetical Mechanisms of Action and Comments on Outcomes. Neuromodulation. 2017 Aug;20(6):525-533 